\title{
Negative Pressure Pulmonary Edema on Extubation of a 9-Month-Old Baby Boy
}

\author{
Dennis E. Feierman, Katerina Svigos, Evan P. Salant, Agnes Miller \\ Department of Anesthesiology, Maimonides Medical Center, New York, USA \\ Email: dfeierman@maimonidesmed.org
}

Received 22 April 2015; accepted 15 May 2015; published 21 May 2015

Copyright (C) 2015 by author and Scientific Research Publishing Inc.

This work is licensed under the Creative Commons Attribution International License (CC BY). http://creativecommons.org/licenses/by/4.0/

(c) (i) 0pen Access

\begin{abstract}
Background: Negative Pressure Pulmonary Edema (NPPE) is an uncommon, but well recognized clinical entity that continues to be reported as a complication of upper airway obstructions during induction or emergence. It results from the negative intrathoracic pressure generated with spontaneous ventilation with concurrent upper airway obstruction. Aim: To present an unusual case of NPPE and review the pathophysiology and treatment. Case: It usually occurs in young healthy athletic adults. We are reporting NPPE in a nine-month-old ex-premature baby. We discuss his intraoperative events leading to NPPE, subsequent intraoperative course and treatment. Conclusion: NPPE needs to be promptly recognized and treated. If the edema resolves, the patient can be successfully extubated, but should be observed overnight.
\end{abstract}

\section{Keywords}

Negative Pressure Pulmonary Edema, Extubation

\section{Introduction}

Negative Pressure Pulmonary Edema (NPPE) is an uncommon complication that can occur during induction of anesthesia, but usually occurs during emergence after extubation. Although NPPE is an uncommon complication, it is still a well recognized clinical entity that results from the negative intrathoracic pressure generated with spontaneous ventilation with concurrent upper airway obstruction [1]. The pulmonary edema results from either "pulling" of fluids from the pulmonary capillary bed into the alveoli or injury to the pulmonary microvascular membranes from severe mechanical stress that results in capillary "leaking" of fluid. NPPE develops after the relief of acute upper airway obstruction caused by epiglottitis, tumors, obesity, hiccups, obstructive sleep apnea, or mediastinal masses [2]. Additionally, it can be caused by laryngospasm during induction or following extubation. Desaturation results from ventilation and perfusion difficulties (V/Q mismatching) and/or shunting. This 
frequently requires reintubation and temporary ventilatory support. Our aim is to briefly review the pathophysiology and our treatment of NPPE.

\section{Case Report}

A 32-week ex-premature baby boy with a history of an uneventful 2-week NICU stay, now 9 months old who presented for first stage hypospadias repair with a buccal graft. Patient was an ASA 2, $7.7 \mathrm{~kg}, 27$ inches with no surgical or past medical history other than prematurity. There was no family history of complication with anesthesia. He had NDKA, was not on any medications and his physical exam was unremarkable with a normal appearing airway.

\subsection{Intraoperative}

Anesthesia was induced via mask with $8 \%$ sevoflurane. After IV cannulation of the left saphenous vein, the patient received $40 \mathrm{mcg}$ ( $5 \mathrm{mcg} / \mathrm{kg}$ ) of fentanyl and $5 \mathrm{mg}$ of rocuronium. The patient was easily intubated with a 3.5 cuffed tube and the cuff was sealed at $25 \mathrm{~cm} \mathrm{H}_{2} \mathrm{O}$. The tube was secured at $12 \mathrm{~cm}$ and the patient had bilateral breath sounds. The case proceeded uneventfully for the next 4 hrs. At the end of the case a caudal block was placed and muscle relaxation was reversed. The patient was fully awake when the ET-tube was removed. The patient coughed out the oral airway and on the next breath went into severe laryngospasm, followed by desaturation. Positive pressure via the mask was initiated while the resident was instructed to push $10 \mathrm{mg}$ succinylcholine and $10 \mathrm{mg}$ propofol along with atropine $120 \mathrm{mcg}$. Despite adequate ventilation on $100 \% \mathrm{O}_{2}$, the saturation did not go above 92\%. The patient was re-intubated with another 3.5 cuffed tube at which point copious amounts of pink froth was coming out of the ET-tube, confirming NPPE. The patient remained intubated in the OR for the next hour, on 6 of PEEP and 60 - $70 \mathrm{ml}$ tidal volume. The ET-tube was suctioned several times during the next hour, and toward the end the froth secretions resolved. His $\mathrm{O}_{2}$ saturations improved significantly.

\subsection{Postoperative Course}

The patient remained intubated in the OR for the next hour, on $6 \mathrm{~cm} \mathrm{H}_{2} \mathrm{O}$ of PEEP and $60-70 \mathrm{ml}$ tidal volume. The ET-tube was suctioned several times during the next hour, and toward the end the froth excretions disappeared and he was spontaneously breathing. He was successfully and uneventfully extubated approximately 1 hour after re-intubation.

He was transferred to the PACU where vital signs remained stable. He was transferred to the PICU for observation. A chest X-ray 5.5 hours after the event was unremarkable. The patient remained stable overnight and $\mathrm{O}_{2}$ saturations were $100 \%$ on room air. The patient was discharged the next day. He returned for the second stage hypospadias repair approximately 6 months later. The second stage hypospadias repair proceeded uneventfully and he was extubated without problems.

\section{Discussion}

The pathogenesis of Negative Pressure Pulmonary Edema is related to the development of high negative intrapleural pressure by vigorous inspiratory efforts against an obstructed upper airway [3]. The etiology and risk factors are listed in Table 1. High negative intrathoracic pressures cause significant fluid shifts from the alveolar capillaries into the alveoli. The negative intrathoracic pressure can result in an increased venous return, which may augment the pulmonary capillary hydrostatic pressures [4]. Additionally, it is believed that there is disruption of the alveolar epithelium and pulmonary capillary membranes, leading to increased permeability and protein-rich pulmonary edema.

The treatment of NPPE on extubation is to maintain a patent upper airway with jaw thrust, CPAP or Pressure Support, and administer supplemental oxygen in order to maintain adequate tissue oxygenation [5]. The differential diagnoses for perioperative hypoxemia are listed in Table 2. In our case, it was easily and quickly recognized. The desaturation was swift, although we were quickly able to adequately ventilate the patient, his saturation remained suboptimal; therefore re-intubation was performed. Analogous to our case, in severe cases re-intubation is required [6]. Ventilation mode should be similar to the mode used during Acute Lung Injury: small tidal volume. Patients should be admitted for observation. These cases usually resolve without additional problems; 
Table 1. Etiology/risk factors for NPPE.

\begin{tabular}{|c|c|}
\hline Etiology & Risk Factors \\
\hline Intraoperative Characteristics & Patient Characteristics \\
\hline Laryngospasm & Young, Healthy, Athletic ${ }^{\mathrm{b}}$ \\
\hline Endotracheal Tube Biting & Obstructive Sleep Apnea \\
\hline Airway Trauma & Surgical Procedures ${ }^{c}$ \\
\hline \multicolumn{2}{|l|}{ Upper Airway Collapse } \\
\hline \multicolumn{2}{|l|}{ Bronchial Obstruction } \\
\hline \multicolumn{2}{|l|}{ Foreign Body Aspiration } \\
\hline \multicolumn{2}{|c|}{ Post-operative Residual Curarization ${ }^{\mathrm{a}}$} \\
\hline \multicolumn{2}{|c|}{$\begin{array}{l}\text { a: Impairs the upper airway dilator muscle strength while preserving inspiratory muscle function; b: Capable of } \\
\text { generating large negative intrathoracic pressures during an obstructive event; c: Oropharyngeal surgery, espe- } \\
\text { cially for tumors or other potentially obstructing masses. }\end{array}$} \\
\hline \multicolumn{2}{|c|}{ Cardiogenic Pulmonary Edema } \\
\hline \multicolumn{2}{|c|}{ Neurogenic Pulmonary Edema } \\
\hline \multicolumn{2}{|c|}{ Fluid Overload } \\
\hline \multicolumn{2}{|c|}{ Acute Respiratory Distress Syndrome } \\
\hline \multicolumn{2}{|c|}{ Anaphylaxis } \\
\hline
\end{tabular}

however, delayed diagnosis can lead to complications [7].

\section{Conclusion}

NPPE needs to be promptly recognized and treated. When no froth is obtained by suctioning the endotracheal tube, the patient can be successfully extubated. However, we recommend observing the patient overnight even if they maintain good oxygenation without desaturation in the post anesthesia care unit.

\section{References}

[1] Krodel, D.J., Bittner, E.A., Abdulnour, R., Brown, R. and Eikermann, M. (2010) Case Scenario: Acute Postoperative Negative Pressure Pulmonary Edema. Anesthesiology, 113, 200-207. http://dx.doi.org/10.1097/aln.0b013e3181e32e68

[2] Hochberg, E.S. (2014) Adult Epiglottitis Complicated by Negative Pressure Pulmonary Edema. Journal of the American Academy of Physician Assistants, 27, 50-52. http://dx.doi.org/10.1097/01.jaa.0000446991.94928.e2

[3] Goldenberg, J.D., Portugal, L.G., Wenig, B.L. and Weingarten, R.T. (1997) Negative-Pressure Pulmonary Edema in the Otolaryngology Patient. Otolaryngology and Head and Neck Surgery, 117, 62-66. http://dx.doi.org/10.1016/S0194-5998(97)70208-0

[4] Fremont, R.D., Kallet, R.H., Matthay, M.A. and Ware, L.B. (2007) Postobstructive Pulmonary Edema: A Case for Hydrostatic Mechanisms. Chest, 131, 1742-1746. http://dx.doi.org/10.1378/chest.06-2934

[5] Rasheed, A., Palaria, U., Rani, D. and Sharma, S. (2014) A Case of Negative Pressure Pulmonary Edema in an Asthmatic Patient after Laparoscopic Cholecystectomy. Anesthesia Essays Research, 8, 86-88. http://dx.doi.org/10.4103/0259-1162.128919

[6] Shigematsu, H., Yoneda, M. and Tanaka, Y. (2014) Negative Pressure Pulmonary Edema Associated with Anterior Cervical Spine Surgery. Asian Spine Journal, 8, 827-830. http://dx.doi.org/10.4184/asj.2014.8.6.827

[7] Kaya, Z., Tuncez, A., Gok, U., Gul, E.E. and Altunbas, G. (2014) Negative Pressure Pulmonary Edema Following Septoplasty Surgery Triggering Acute Subendocardial Myocardial Infarction. Heart Views, 15, 46-48. http://dx.doi.org/10.4103/1995-705X.137495 